5.2 Myocardial Integrated Backscatter in Normotensive and Hypertensive

\title{
Diabetic Patients
}

A.C.M. Capra (1), C. Giannattasio (1), L. Viscardi (1), F. Soriano (1),

F. Papalettera (2), P. Canova (1), P. Campadello (1), D. Dozio (1), A. Cafro (1),

F. Paleari (2), G. Mancia (1)

(1)Università Milano - Bicocca, Monza; (2)Ospedale San Gerardo, Monza, Italy Introduction. Diabetes is often associated to the so-called 'diabetic cardiomiopathy'.

Aim. To investigate cardiac tissue characterization, that is cardiac fibrosis, using dedicated software for integrated backscatter analysis, which provides information on cardiac tissue composition even in absence of obvious cardiac systolic and diastolic dysfunction at a standard echo examination.

Methods. We studied 30 subjects. 22 had a type 2 uncomplicated diabetes, 12 of which were normotensive (group A) and 10 hypertensive (group B) patients, whereas the remaining 8 subjects were healthy age-matched controls (group C). All diabetics assumed oral antidiabetic drugs and al hypertensives an ACE inhibitor. In each subject we performed a standard echo evaluating indexed left ventricular mass and systo-diastolic function: cardiac tissue characterization by integrated backscatter analysis was expressed as mean value (average IBS) and systo-diastolic or cyclic variation.

Results. Blood pressure progressively increased from group C to group A and B (121/68 $\pm 6.5 ; 133 / 71$

$\pm 7.2 ; 145 / 90 \pm 12.5 \mathrm{mmHg}$ ). Left ventricular mass index and indices of systo-diastolic function were similar in the 3 groups, except for the E/A ratio which had the lowest value in diabetic hypertensive patients (group B) compared to the other two groups ( 0.6 vs 0.9 in group $\mathrm{A}$ and $\mathrm{C}, \mathrm{p}<0.05)$. There was from $C$ to $A$ and $B$ a progressive increase in the average IBS value (group C: $20.5 \pm 2.6$, group $A$ : 21.4 \pm 1.7 , group $\mathrm{B}: 25.7 \pm 1.3 \mathrm{~dB}, \mathrm{p}<0.05$ ) as well as a progressive reduction in cyclic variation (group C: $9.6 \pm 1.8$, group A: $7.7 \pm 1.1$, group B: $5.5 \pm 0.5$, p $<0.05$ ).

Conclusions. Even with a normal echocardiographic standard evaluation diabetics show higher values of average backscatter and lower values of cyclic variation: such data may suggest a more abundant presence of fibrosis and consequently early structural impairment which is more evident when diabetes is associated to high blood pressure. 